\title{
Sentinel lymph node biopsy for conjunctival malignant melanoma: surgical techniques
}

\author{
This article was published in the following Dove Press journal: \\ Clinical Ophthalmology \\ 16 December 2014 \\ Number of times this article has been viewed
}

\author{
Alberto JA Wainstein ${ }^{1,2}$ \\ Ana P Drummond-Lage' \\ Milhem JM Kansaon ${ }^{2}$ \\ Gustavo O Bretas ${ }^{2}$ \\ Rodrigo F Almeida ${ }^{3}$ \\ Ana LF Gloria ${ }^{3}$ \\ Ana RP Figueiredo ${ }^{3}$
}

'Faculty of Medical Sciences of Minas Gerais, ${ }^{2}$ Oncad Surgical Oncology, ${ }^{3}$ Ophthalmology Department, Federal University of Minas Gerais, Belo

Horizonte, Brazil
Correspondence: Alberto JA Wainstein Faculdade de Ciências Médicas de Minas Gerais, Alameda Ezequiel Dias, 275, $2^{\circ}$ Andar, CEP 30I30-II 0 Belo Horizonte, MG Brazil

Tel +553 3I 3022 II 100

$\mathrm{Fax}+553132247545$

Email albertojaw@gmail.com
Background: The purpose of this report is to examine the viability and safety of preoperative lymphoscintigraphy and radio guided sentinel lymph node (SLN) biopsy for conjunctival melanoma, and to identify the best technique to perform this procedure.

Methods: Three patients diagnosed with malignant melanoma of the conjunctiva underwent lymphoscintigraphy and SLN biopsy using a dual technique comprising isosulfan blue dye and technetium Tc 99m sulfur colloid. Each patient was anesthetized and the conjunctival melanoma was excised. SLNs were localized by a gamma probe, identified according to radioactivity and sentinel blue printing, and dissected, along with drainage of the associated lymphatic basins. The SLNs were evaluated by a pathologist using hematoxylin-eosin staining following serial sectioning and immunohistochemistry using a triple melanoma cocktail (S-100, Melan-A, and HMB-45 antigens).

Results: Two SLNs were stained in the jugular chain during preoperative lymphoscintigraphy in the first patient, two SLNs were identified in the preauricular and submandibular areas in the second patient, and two SLNs were identified in the submandibular and parotid areas in the third patient. All lymph nodes identified by lymphoscintigraphy were dissected and identified at surgery with $100 \%$ accuracy in all three patients. All SLNs were histologically and immunohistochemically negative. Patients had good cosmetic and functional results, and maintained their visual acuity and ocular motility.

Conclusion: Patients with conjunctival melanoma can undergo preoperative lymphoscintigraphy and SLN biopsy safely using radioactive technetium and isosulfan blue dye.

Keywords: ocular melanoma, sentinel lymph node biopsy, lymphoscintigraphy, conjunctival melanoma

\section{Introduction}

Conjunctival melanoma occurs approximately $40 \%$ less often than choroidal melanoma. It is a rare tumor, accounting for less than $1 \%$ of all malignant eye tumors, but has a relatively high mortality rate of up to $20 \%$ in 10 years. It is identified most frequently in the perilimbal interpalpebral bulbar conjunctiva, with tumors located in the palpebral or forniceal conjunctiva or caruncle, plica semilunaris, or eyelid margins having a worse prognosis. ${ }^{1}$ It is characterized by a perceptible atypical pigmented tumor of varying color in any region of the conjunctiva, which may spread initially into the lymphatic system via a sentinel lymph node (SLN). As for most malignant tumors, the best treatment for conjunctival melanoma is complete excision of the lesion with wide free margins using the "no-touch" technique of cryotherapy (double freezethaw). Cryotherapy at the epithelium should be done using absolute alcohol to avoid the event of corneal injury.

Factors influencing the prognosis of conjunctival melanoma include location of the primary lesion, tumor thickness, ulceration, mitotic index, lymph node metastasis, 
and distant metastasis. The presence of regional lymph node metastasis is one of the most important predictors of recurrence in patients with conjunctival melanoma.

The rationale for SLN biopsy is that there is a logical pattern of disease progression via lymphatic drainage of a cancer cell to the regional lymph node basin, and that the first lymph node, ie, the SLN, is the initial barrier to further spread of tumor cells. ${ }^{2}$

Accepted as the standard of care for melanoma and breast cancer in particular, SLN biopsy concept supports that tumor cells spread via SLN, and the technique is very useful for staging of certain types of solid tumor. SLN biopsy has a lower cost and less morbidity when compared with elective or prophylactic radical lymphadenectomy. SLN biopsy is a relatively standard and well established technique, and is recommended by Cohen at $\mathrm{al}^{3}$ for conjunctival melanoma. Importantly, their paper provides data supporting use of this approach for conjunctival melanoma in clinical practice.

Herein, we present procedure-related data for three patients with rare conjunctival melanoma who underwent tumor resection with SLN biopsy at our institution, where we have established routines and a methodology for use of SLN biopsy in ocular melanoma.

\section{Materials and methods}

We report a series of three patients with conjunctival melanoma who underwent SLN biopsy in our department. In patients with conjunctival melanoma, there is a group at high risk of regional or distal recurrence. One of the main selection criteria for SLN biopsy in this study was conjunctival melanoma thicker than $2 \mathrm{~mm}$ as determined by histologic analysis. This eligibility criterion can identify patients who should be better staged with SLN biopsy regarding a worse prognosis. ${ }^{3,4}$ Is important to select patients in order to evaluate adjuvant treatment and where possible to establish strict follow-up to identify and treat metastatic disease with target therapies or immunoactive drugs.

Preoperative lymphoscintigraphy is performed prior to surgery. On the day of surgery, a $0.2 \mathrm{~mL}$ volume of an activity $10 \mathrm{MBq}(0.3 \mathrm{mCi})$ of technetium Tc $99 \mathrm{~m}$ sulfur colloid is injected into the subconjunctival space around the eyelid lesion. ${ }^{5}$ We recommend this concentrated radioactivity in a small volume to avoid local changes at the surgical site. Each patient remains in the nuclear medicine department for serial lymphoscintigraphy (Figure 1) until localization of SLN. This technique affords excellent functional analysis but has limited anatomic resolution, so the best option is to

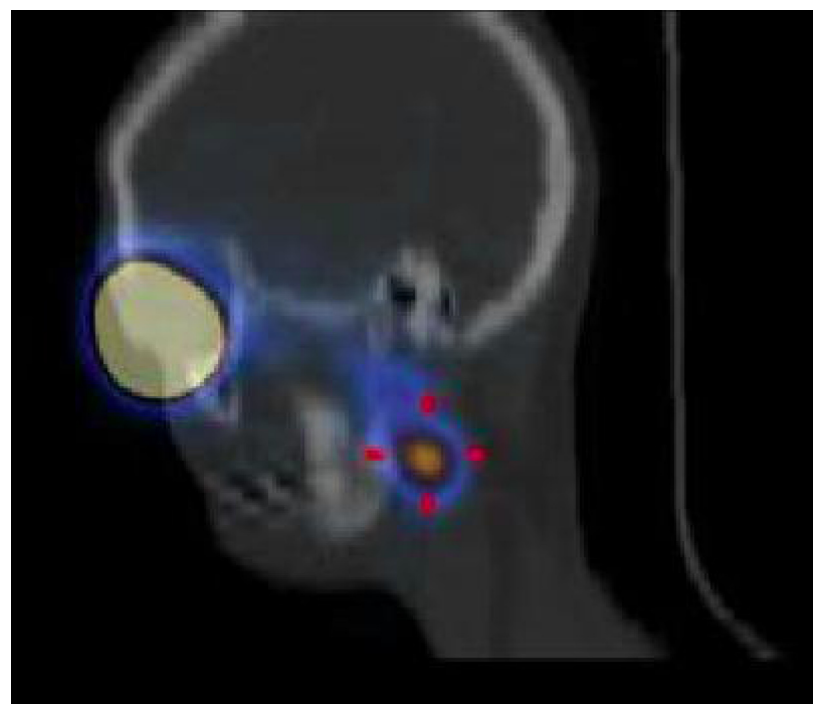

Figure I Single photon emission computed tomography/computed tomography demonstrating a submandibular sentinel lymph node (red arrows) with its anatomical references in a patient (case 3 ) with melanoma of the conjunctiva.

Note: The white area indicates the original injection site.

use single photon emission computed tomography/computed tomography as part of lymphoscintigraphy. This is a good example of the merging of high functional resolution of nuclear medicine images with the high anatomic resolution of computed tomography. This technique accurately confirms the SLN and also its anatomic position. ${ }^{5}$

Patients are taken to theater and receive local anesthesia plus venous sedation. An extra volume of isosulfan blue dye is administered around the tumor area to mark the SLN. ${ }^{6}$ Using this double technique, a radioactive blue lymph node can be achieved that facilitates localization and resection with less morbidity and a small scar. The primary tumor is resected according to best practice to achieve an R0 oncologic result (Figure 2). It is also important starting removing primary lesion to take out the radioactivity background that usually difficulty the identification of SLN.

A handheld gamma probe is used to identify SLNs transcutaneously, usually after 30 minutes to 4 hours considering the injection of technetium Tc $99 \mathrm{~m}$ sulfur colloid as the time zero. A small $(1-3 \mathrm{~cm})$ incision is made over each area of increased radioactivity. ${ }^{6}$ Each identified SLN is dissected carefully under color and radioactivity guidance. All SLN are sent for pathologic analysis according to the following parameters: radioactive and blue-labeled, radioactive and not labeled, blue-labeled and not radioactive. During SLN dissection, all lymph nodes identified as suspicious are also removed and labeled as non-SLNs. All lymph nodes removed and submitted for histopathology evaluation are first examined by hematoxylin eosin staining. If there is no evidence of 

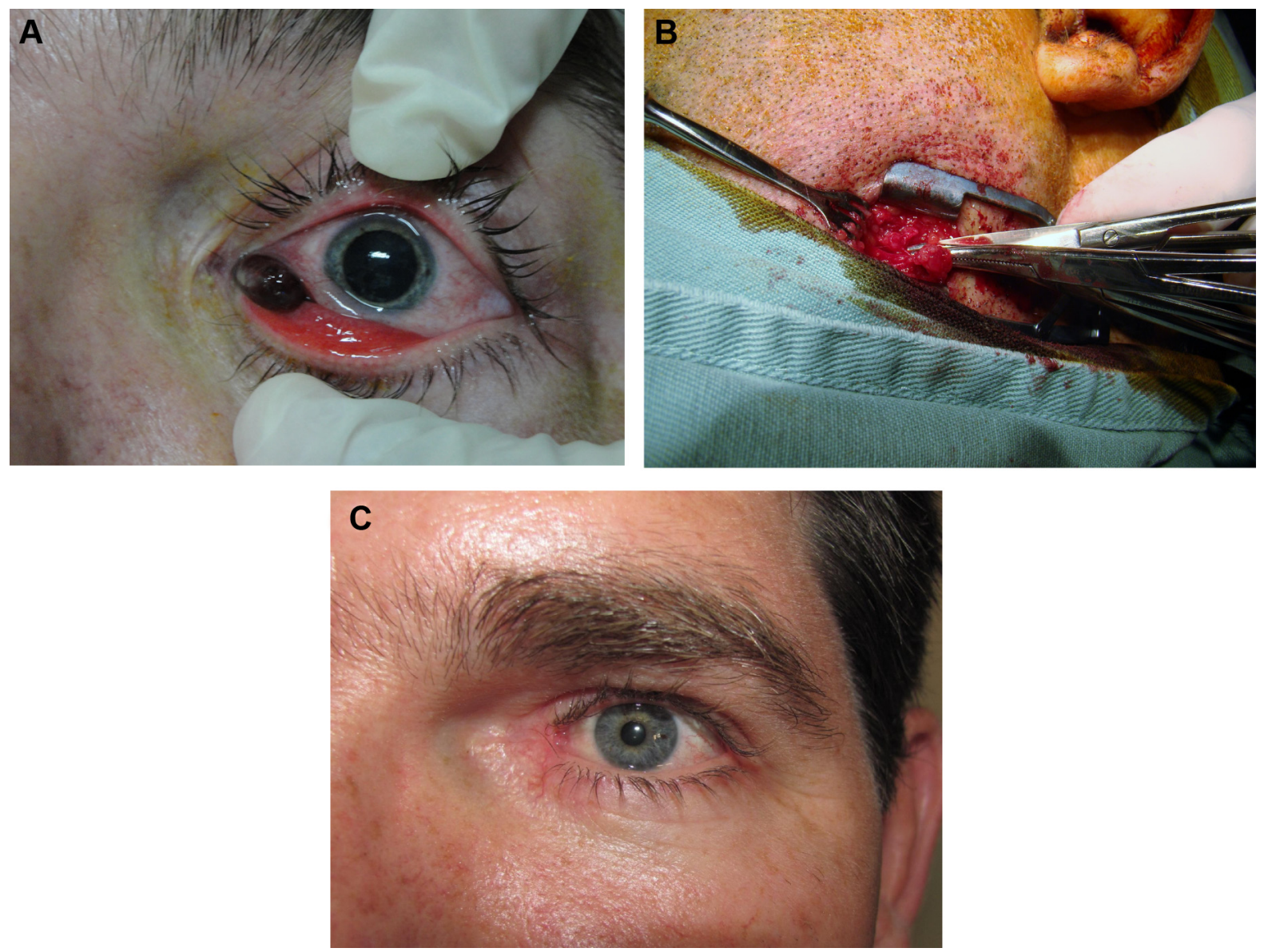

Figure 2 Conjunctival melanoma before and after excision (case 3). (A) Excision of the lesion. (B) Identification of the submandibular lymph node. (C) Postoperative result.

metastatic disease, this is followed by immunohistochemistry analysis using three glycoproteins, ie, S-100, Melan-A, and HMB-45 antigen, for more accurate results. Patients with metastatic SLN are offered radical lymphadenectomy of the metastatic lymph node chain as a standard procedure. For patients with advanced regional disease, ie, N2 or N3, we discuss adjuvant radiotherapy.

We now present a description of three cases of rare conjunctival melanoma and the respective surgical techniques used, including oncologic R0 resection and double marker SLN biopsy. All patients were male, had a previous diagnosis of conjunctival melanoma on incisional biopsy, and had no signs of distant metastasis (stage II disease). They opted for curative local oncologic R0 excision plus regional microscopic staging by SLN biopsy.

\section{Case I}

A 74-year-old man was diagnosed to have melanoma of the left medial and lateral lower eyelid with conjunctival invasion at our institution in January 2007. There were no signs of distant disease. Lymphoscintigraphy performed on the morning of surgery identified two SLN, one submandibular and one in the left parotid area. Both lymph nodes were localized using a gamma probe and removed surgically with a minimal incision. Surgical intervention was performed, expanding the margins oncologically with wide excision of the lower tarsal conjunctiva, medial upper tarsal conjunctiva, and tarsal margin of temporal lobe. The pathology report showed no tumor in the conjunctiva margins and all SLN to be free of metastatic melanoma on hematoxylin-eosin staining, which was confirmed by immunohistochemistry. The patient was staged as T3N0M0 (stage II disease). The patient developed a lung metastasis in June 2008.

\section{Case 2}

A 40-year-old man attended our institution in February 2010 complaining of a pigmented lesion in the medial corner of the left eye. He reported that he underwent excision of a small pigmented lesion in the same location in 1999, and pathology had suggested a nevus. The lesion reappeared in 2005, 
and had grown rapidly in the previous 2 months. Ophthalmic examination revealed visual acuity of 20/20, an intraocular pressure of $14 \mathrm{mmHg}$ in both eyes, and normal funduscopy. Biomicroscopy showed the presence of a pigmented tumor at the caruncle, extending to the lower conjunctival fornix and establishing a mass in this region. A computed tomography scan of the orbit showed localization of the tumor in the medial corner without orbital extension.

The patient underwent incisional biopsy of the lesion located in the inferior fornix. Histopathologic examination showed an atypical neoplastic melanocytic lesion in the conjunctiva compatible with ulcerated and invasive melanoma, with thickness of $4.5 \mathrm{~mm}$. There was no evidence of tumor spread. In May 2010, the patient was brought to the nuclear medicine service where Tc-99 was injected in the pericicatricial conjunctiva. After 2 hours, lymphoscintigraphy identified two SLN in the pre-auricular region. Tumor excision was performed with wide margins, achieving an oncologic R0 result. The corner of the eye, the medial third of the upper and lower eyelids, and the lacrimal sac were also excised in monoblock. Reconstruction was done with skin graft. At the beginning of the procedure, isosulfan blue dye was injected into the scar in order to stain the SLN and help in identification of the surgical field. The oncology surgeons localized and resected the two previously identified nodes using a gamma probe intraoperatively. The lymph nodes were processed using the "bread loaf" technique in $2 \mathrm{~mm}$ thick slices. Hematoxylineosin-stained (HE) sections from all blocks of the primary lesion were analyzed initially, confirming the diagnosis of melanoma. At SLN biospsy, clear margins and SLN were negative for metastasis with Hematoxylin-eosin-stained. All SLN initially negative at HE demanded a confirmatory immunohistochemistry using a triple antibody cocktail (HMB-45, S-100, Melan A) and were considered free of metastasis if they were negative for all three markers.

After confirmation of clear margins, reconstruction of the medial corner of the eye, fornices, and ocular surface was performed using an oral mucosa graft and amniotic membrane transplantation. The patient had a good cosmetic and functional result, maintaining his visual acuity and ocular motility, and has been followed up since then with no signs of disease recurrence. The patient was staged as T4N0M0 (stage II disease). Because of his negative SLN biopsy, the patient was spared the morbidity of radical lymph node dissection.

\section{Case 3}

A 27-year-old man presented to our institution in May 2010 with a diagnosis of melanoma in the temporal bulbar conjunctiva of the left eye, after have been operated on in another institution in the previous February. The pathology report described malignant melanocytic conjunctival mucosa/invasive nodular melanoma (Breslow thickness $3 \mathrm{~mm}$, Clark level IV), with deeply compromised margins. Ophthalmologic examination revealed a visual acuity of 20/20, an intraocular pressure of $14 \mathrm{mmHg}$ in both eyes, and normal funduscopy. There were no signs of distant disease. Surgery was performed to expand the oncologic margins, with wide excision of the entire temporal bulbar conjunctiva, including lamellar sclerectomy continuously to the insertion of lateral rectus muscle, conjunctival cryotherapy, and finally SLN biopsy. Lymphoscintigraphy performed on the same day as the surgery identified two SLN, one preauricular and the other submandibular. Both lymph nodes were localized using a gamma probe and removed surgically with minimal incision. Reconstruction was performed using amniotic membrane implantation. The pathology report indicated no tumor at the conjunctiva margins and sclera. Both SLN were free of metastatic melanoma by hematoxylin-eosin staining, which was confirmed by immunohistochemistry. The patient had a good cosmetic and functional result, maintaining his visual acuity and ocular motility, and has been followed since with no signs of disease recurrence. The patient was staged as T3N0M0 (stage II disease).

\section{Discussion}

Conjunctival melanoma occurs most often in the fifth and sixth decades of life, with no gender predilection, and predominantly affects Caucasians. It is a tumor with highly variable pigmentation in the conjunctival and interpalpebral perilimbal areas. ${ }^{7}$ However, it may also present in any conjunctival region as a non-pigmented lesion.

The etiology of conjunctival melanoma is controversial. According to Noregard et al, $36 \%$ of conjunctival melanomas are derived from primary acquired melanosis, $16 \%$ from nevi, and $47 \%$ arise de novo. ${ }^{8}$ Recent findings presented by Shields et al indicate that $75 \%$ of melanomas are derived from primary acquired melanosis, 20\% from nevi, and 5\% de novo. ${ }^{9}$

In rare cases, conjunctival melanoma can invade the globe or ocular orbit. The usual site of metastasis is a lymph node (in $45 \%-60 \%$ of patients with regional metastasis), mainly in the intraparotid and submandibular areas. Other common sites of metastasis are the brain, lung, liver, bone, skin, and gastrointestinal tract. The thickness of the lesion is measured by the distance between the epithelial surface and 
the deeper tissue where is seen the presence of neoplastic cells. Thickness is an important prognostic factor. Unlike in patients with skin melanoma, prophylactic lymph node radical dissection has not been performed in patients with conjunctival melanoma. Further, it has taken some time for SLN biopsy, which saves many patients with skin melanoma from undergoing unnecessary surgery, to be regularly offered to patients with conjunctival melanoma.

The risk factors for metastasis are incomplete excision (positive margins), ulceration, a high mitotic index, and lesion thickness. Five-year survival is approximately $80 \%$ for patients with tumors less than $4 \mathrm{~mm}$, but decreases to $55 \%$ for tumors deeper than $4 \mathrm{~mm}$. The cutoff for high risk versus low risk for recurrence of conjunctival melanoma is considered to be $2 \mathrm{~mm}^{3}$

In the past, some melanoma services had advocated prophylactic lymph node dissection to try to control lymphatic dissemination. Despite attempts to improve disease-free survival and cure rates, this practice has not been taken up because of its high morbidity and the fact that there is no unique pattern of lymphatic drainage from the conjunctiva. Drainage to the parotid, submandibular, and occipital regions and also to many cervical areas makes it impossible to predict which lymphatic site should be cleared prophylactically.

SLN biopsy for conjunctival melanoma was first reported in 2001. Since then, various reports have been published regarding the use of the procedure for eyelid or conjunctival melanoma. ${ }^{2,3,10,11}$ Application of SLN biopsy techniques for conjunctival melanoma leads to improved staging and prognostication. Patients with lymph nodes free of neoplastic disease (stage II) have longer survival than those with regionally metastatic disease (stage III). Those with positive lymph nodes are candidates for regional lymphadenectomy of the lymphatic chain affected and also adjuvant radiotherapy in selected cases. Patients with metastatic disease must be evaluated for systemic chemotherapy or immunotherapy.

The disadvantage of SLN biopsy is the risk of a false negative result, which could be substantially decreased with training of surgical teams. Surgery to the head and neck represents a major challenge to the surgeon dealing with these patients. The technical and anatomic aspects involved in this type of surgery make it very complex to perform preoperatively and postoperatively, demanding specialized surgeons trained in this field. The head and neck area has multifaceted lymphatic drainage when compared with other body regions. The submandibular and parotid nodes are located in areas with loose connective tissue, with fat tissue obscuring neural tissue, what can increase the risk of nerve damage. SLN biopsy can identify one or more SLN, allowing accurate pathologic examination. Precise examination of the specimen and immunohistochemistry assessment using antibodies against S-100, HMB-45, and Melan-A can stage a lymph node correctly. ${ }^{12}$

Although a radical lymphadenectomy can provide large specimens from the many lymph nodes found in a mass of fat tissue, SLN biopsy has the advantage that all the information is concentrated in one or a few lymph nodes, which can be processed and examined by the pathologist using more precise techniques like "bread loaf" slim cuts.

Patients with ocular melanoma, including conjunctival melanoma, have an increased risk of the disease arising in a dysplastic skin nevus, so both patients and their first-degree relatives should be followed by a dermatologist.

Although conjunctival melanoma is a rare tumor, due to its high mortality rate, its correct treatment should be known to all professionals likely to handle patients with this type of disease, including ophthalmologists, oncology surgeons, medical oncologists, and radiation oncologists. Inappropriate treatment can result in local and systemic relapse, invasive surgery with a high morbidity rate, and even death. Any atypical ocular lesion should be considered for biopsy, since many such lesions are precancerous.

SLN biopsy in conjunctival melanoma has an important role in establishing the correct treatment and defining the prognosis. It permits accurate assessment of $\mathrm{N}$ (lymph node metastasis) in the TNM staging system, which is critical for determining the optimal therapeutic modality after surgery.

Patients with melanoma have many options for adjuvant treatment to prevent recurrence and even therapeutic treatment to control metastasis. Precise staging is key to identifying the best approach for the individual patient. SLN biopsy is a very important step in this process, including in patients with conjunctival melanoma. Finally, although preliminary results from a prospective clinical trial evaluating the impact of SLN biopsy on overall survival did not show improvement, ${ }^{13}$ it is clear that SLN biopsy decreased local recurrence, which has a significant impact on quality of life in patients with head and neck melanoma. We are still awaiting the long-term results of MSLT-1 (the Multicenter Selective Lymphadenectomy Trial-1) and also an ongoing MSLT-2 trial, but there is a consensus that SLN biopsy is standard of care for melanoma patients at high risk of recurrence, including conjunctival melanoma, as shown in this paper. 


\section{Disclosure}

The authors report no conflict of interest in this work. No financial support was received for this submission.

\section{References}

1. Seregard S. Conjunctival melanoma. Surv Ophthalmol. 1998;42: 321-350.

2. Zaknun JJ, Giammarile F, Olmos RA, Vidal-Sicart S, Mariani G. Changing paradigms in radioguided surgery and intraoperative imaging: the GOSTT concept. Eur J Nucl Med Mol Imaging. 2012;39:1-3.

3. Cohen VM, Tsimpida M, Hungerford JL, Jan H, Cerio R, Moir G. Prospective study of sentinel lymph node biopsy for conjunctival melanoma. Br J Ophthalmol. 2013;97:1525-1259.

4. Lim LA, Madigan MC, Conway RM. Conjunctival melanoma: a review of conceptual and treatment advances. Clin Ophthalmol. 2013;7: $521-531$.

5. Uren RF. SPECT/CT lymphoscintigraphy to locate the sentinel lymph node in patients with melanoma. Ann Surg Oncol. 2009;16:1459-1460.

6. Ho VH, Ross MI, Prieto VG, Khaleeq A, Kim S, Esmaeli B. Sentinel lymph node biopsy for sebaceous cell carcinoma and melanoma of the ocular adnexa. Arch Otolaryngol Head Neck Surg. 2007;133:820-826.

7. Esmaeli B, Eicher S, Popp J, Delpassand E, Prieto VG, Gershenwald JE. Sentinel lymph node biopsy for conjunctival melanoma. Ophthal Plast Reconstr Surg. 2001;17:436-442.
8. Noregard JC, Gerner N, Jensen OA, Prause JU. Malignant melanoma of the conjunctiva: occurrence and survival following surgery and radiotherapy in a Danish population. Graefes Arch Clin Exp Ophthalmol. 1996;239:569-572.

9. Shields CL, Markowitz JS, Belinsky I, et al. Conjunctival melanoma: outcomes based on tumor origin in 382 consecutive cases. Ophthalmology. 2011;118:385-389.

10. Amato M, Esmaeli B, Ahmadi MA, et al. Feasibility of preoperative lymphoscintigraphy for identification of sentinel lymph nodes in patients with conjunctival and periocular skin malignancies. Ophthal Plast Reconstr Surg. 2003;19:102-106.

11. Savar A, Ross MI, Prieto VG et al. Metastasis in SLN indicates the initial spread from a primary tumor and its biopsy is commonly applied as staging procedure. Esmaeli B. Sentinel lymph node biopsy for ocular adnexal melanoma: experience in 30 patients. Ophthalmology. 2009;16:2217-2223.

12. Prieto VG, Clark SH. Processing of sentinel lymph nodes for detection of metastatic melanoma. Ann Diagn Pathol. 2002;6:257-264.

13. Morton DL, Thompson JF, Cochran AJ, et al. Final trial report of sentinel-node biopsy versus nodal observation in melanoma. $N$ Engl $J$ Med. 2014;370:599-609.
Clinical Ophthalmology

\section{Publish your work in this journal}

Clinical Ophthalmology is an international, peer-reviewed journal covering all subspecialties within ophthalmology. Key topics include: Optometry; Visual science; Pharmacology and drug therapy in eye diseases; Basic Sciences; Primary and Secondary eye care; Patient Safety and Quality of Care Improvements. This journal is indexed on

\section{Dovepress}

PubMed Central and CAS, and is the official journal of The Society of Clinical Ophthalmology (SCO). The manuscript management system is completely online and includes a very quick and fair peer-review system, which is all easy to use. Visit http://www.dovepress.com/ testimonials.php to read real quotes from published authors. 